\title{
A combinatorial analysis of Severi degrees
}

\author{
$\mathrm{Fu}$ Liu战 \\ ${ }^{1}$ University of California, Davis, CA, USA.
}

\begin{abstract}
Based on results by Brugallé and Mikhalkin, Fomin and Mikhalkin give formulas for computing classical Severi degrees $N^{d, \delta}$ using long-edge graphs. In 2012, Block, Colley and Kennedy considered the logarithmic version of a special function associated to long-edge graphs which appeared in Fomin-Mikhalkin's formula, and conjectured it to be linear. They have since proved their conjecture. At the same time, motivated by their conjecture, we consider a special multivariate function associated to long-edge graphs that generalizes their function. The main result of this paper is that the multivariate function we define is always linear.
\end{abstract}

The first application of our linearity result is that by applying it to classical Severi degrees, we recover quadraticity of $Q^{d, \delta}$ and a bound $\delta$ for the threshold of polynomiality of $N^{d, \delta}$. Next, in joint work with Osserman, we apply the linearity result to a special family of toric surfaces and obtain universal polynomial results having connections to the Göttsche-Yau-Zaslow formula. As a result, we provide combinatorial formulas for the two unidentified power series $B_{1}(q)$ and $B_{2}(q)$ appearing in the Göttsche-Yau-Zaslow formula.

The proof of our linearity result is completely combinatorial. We define $\tau$-graphs which generalize long-edge graphs, and a closely related family of combinatorial objects we call $(\boldsymbol{\tau}, \boldsymbol{n})$-words. By introducing height functions and a concept of irreducibility, we describe ways to decompose certain families of $(\boldsymbol{\tau}, \boldsymbol{n})$-words into irreducible words, which leads to the desired results.

Résumé. Basé sur les travaux de Brugallé et Mikhalkin, Fomin et Mikhalkin ont donné des formules pour calculer les degrés de Severi classiques $N^{d, \delta}$ en utilisant des graphes aux arêtes longues. En 2012, Block, Colley et Kennedy ont considéré la version logarithmique d'une fonction spéciale associée aux graphes aux arêtes longues qui apparait dans la formule de Fomin et Mikhalkin, et ont conjecturé qu'elle est linéaire. Ils ont depuis montré leur conjecture. Au même moment, motivés par leur conjecture, nous avons considéré une fonction spéciale multivariée associée aux graphes aux arêtes longues qui généralise leur fonction. Le résultat principal de cet article est que la fonction multivariée que nous définissons est toujours linéaire.

La première application de notre résultat de linéarité est qu'en l'appliquant aux degrés de Severi classiques, nous retrouvons le fait que $Q^{d, \delta}$ est quadratique et une borne $\delta$ pour le seuil de polynomialité de $N^{d, \delta}$. Ensuite, dans un travail en commun avec Osserman, nous appliquons le résultat de linéarité pour une famille particulière de surfaces toriques et obtenons des résultats sur les polynômes universels ayant des connexions avec la formule de Göttsche, Yau et Zaslow. En conséquence, nous obtenons des formules combinatoires pour deux séries formelles $B_{1}(q)$ et $B_{2}(q)$ non-identifiées apparaissant dans la formule de Göttsche, Yau et Zaslow.

La preuve de notre résultat de linéarité est purement combinatoire. Nous définissons des $\tau$-graphes qui généralisent les graphes aux arêtes longues, une famille étroitement liée d'objets combinatoires que nous appelons les $(\boldsymbol{\tau}, \boldsymbol{n})$-mots. En introduisant des fonctions de hauteur et un concept d'irreductibilité, nous décrivons des façons de décomposer certaines familles de $(\boldsymbol{\tau}, \boldsymbol{n})$-mots en mots irreductibles, ce qui nous conduit aux résultats souhaités.

\footnotetext{
${ }^{\dagger}$ Fu Liu is partially supported by NSF grant DMS-1265702. 
Keywords. Severi degree, long-edge graph, $\boldsymbol{\tau}$-graph, $(\boldsymbol{\tau}, \boldsymbol{n})$-word

\section{Background on Severi degrees}

The classical Severi degree, denoted by $N^{d, \delta}$, is the degree of the Severi variety. It counts the number of curves of degree $d$ with $\delta$ nodes passing through $\frac{d(d+3)}{2}-\delta$ general points in the complex projective plane $\mathbb{C P}^{2}$. If $d \geq \delta+2$, the Severi degree $N^{d, \delta}$ coincides with the Gromov-Witten invariant $N_{d, \frac{(d-1)(d-2)}{2}-\delta}$, which counts maps from curves to the plane. The problem of studying the Severi degrees dates back to late 19th century by Chasles, Zeuthen and Schubert. The modern study of Severi varieties was initiated by Harris' proof of their irreducibility [9].

In 1994, Di Francesco and Itzykson [6] conjectured that for fixed $\delta$, the Severi degree $N^{d, \delta}$ is given by a node polynomial $N_{\delta}(d)$ for sufficiently large $d$. In 2009, Fomin and Mikhalkin [7, Theorem 5.1] established the polynomiality of $N^{d, \delta}$ using tropical geometry and floor decomposition. Since then Block has computed the node polynomial $N_{\delta}(d)$ up to $\delta=14$ [1]. The threshold of the polynomiality of $N^{d, \delta}$ is the value $d^{*}=d^{*}(\delta)$ such that $N^{d, \delta}=N_{\delta}(d)$ for all $d \geq d^{*}$. Fomin and Mikhalkin [7] showed that $d^{*} \leq 2 \delta$; Block [1] lowered it to $d^{*} \leq \delta$; and most recently Kleiman and Shende [10] proved the bound $d^{*} \leq\lceil\delta / 2\rceil+1$ conjectured by Göttsche.

Instead of restricting the attention to $\mathbb{C P}^{2}$, one can ask the same question of enumerating curves on other surfaces. Let $L$ be a line bundle on a complex projective smooth surface $S$. We denote by $N^{\delta}(S, L)$ the number of $\delta$-nodal curves in $|L|$ passing through $\operatorname{dim}|L|-\delta$ points in general position. When $S=\mathbb{C P}^{2}$ and $L=\mathcal{O}_{\mathbb{C P}^{2}}(d)$, we recover the classical Severi degree $N^{d, \delta}$. Hence, we can consider $N^{\delta}(S, L)$ to be a generalized Severi degree. In [8, Conjecture 2.1], Göttsche conjectured that for every $\delta$, there exists a universal polynomial $T_{\delta}(x, y, z, w)$ of degree $\delta$ that computes the numbers $N^{\delta}(S, L)$ by evaluating $T_{\delta}$ at the four topological numbers of $(S, L): L^{2}, L K_{S}, K_{S}^{2}$ and $c_{2}(S)$, provided that the line bundle $L$ is $(5 \delta-1)$-very ample. Furthermore, inspired by the Yau-Zaslow formula, Göttsche [8, Conjecture 2.4] conjectured the closed form of the generating function of $T_{\delta}$, which is known as the Göttsche-YauZaslow formula. Recently, Tzeng [15] and Kool-Shende-Thomas [11] independently proved Göttsche's conjectures. Note that in the case of $\mathbb{C P}^{2}$, the four topological numbers become: $L^{2}=d^{2}, L K_{S}=$ $-3 d, K_{S}^{2}=9$ and $c_{2}(S)=3$. Thus,

$$
T_{\delta}\left(d^{2},-3 d, 9,3\right)=N_{\delta}(d)
$$

In [8], Göttsche discussed a consequence of the Göttsche-Yau-Zaslow’s formula.

Proposition 1.1 ([8], Proposition 2.3) There exist four universal power series $A_{1}(t), A_{2}(t), A_{3}(t)$ and $A_{4}(t)$ such that

$$
\log \left(\sum_{\delta \geq 0} T_{\delta}(x, y, z, w) t^{\delta}\right)=x A_{1}(t)+y A_{2}(t)+z A_{3}(t)+w A_{4}(t) .
$$

This means that the coefficient of $t^{\delta}$ in the formal logarithm of $\sum_{\delta \geq 0} T_{\delta}(x, y, z, w) t^{\delta}$ is a linear function in $x, y, z$ and $w$, which is potentially simpler than the expression for $T_{\delta}(x, y, z, w)$.

Therefore, it is natural for us to consider the generating function for classical Severi degrees and its formal logarithm:

$$
\mathcal{N}(d):=1+\sum_{\delta \geq 1} N^{d, \delta} t^{\delta}, \quad \mathcal{Q}(d):=\log (\mathcal{N}(d))=\sum_{\delta \geq 1} Q^{d, \delta} t^{\delta} .
$$


It is straightforward to show that $Q^{d, \delta}$ is also a polynomial in $d$ for sufficiently large $d$. We denote this polynomial by $Q_{\delta}(d)$. It is clear that

$$
\log \left(\sum_{\delta \geq 0} N_{\delta}(d) t^{\delta}\right)=\sum_{\delta \geq 1} Q_{\delta}(d) t^{\delta} .
$$

Although the degree of $N_{\delta}(d)$ was shown to be $2 \delta$, the polynomial $Q_{\delta}(d)$, which is an alternating sum of $N_{\delta}(d)$ 's, turns out to be quadratic, following from $(1.1)$ and Proposition 1.1. (See Proposition 3.1 in [13].)

Corollary 1.2 For any fixed $\delta, Q^{d, \delta}$ is a quadratic polynomial in d for sufficiently large $d$.

In this paper, we prove a certain function associated to long-edge graphs is linear. Using this result, we provide another proof of Corollary 1.2 as well as a combinatorial way of computing the power series $A_{1}(t)$ and $A_{2}(t)$ of Proposition 1.1. Further, in joint work with Osserman [12], the linearity result enables us to obtain a universal polynomiality property of Severi degrees on families of toric surfaces.

In the next section, we give a brief introduction to the objects in our results, and state the main theorem of the paper.

\section{Long-edge graphs and the main result}

Brugallé and Mikhalkin [3, 4] introduced “(marked) labeled floor diagrams" and gave an enumerative formula for the Severi degree $N^{d, \delta}$ in terms of these diagrams. Fomin and Mikhalkin [7] reformulated Brugallé and Mikhalkin's results by introducing a "template decomposition" of labeled floor diagrams. They first constructed a bijection between labeled floor diagrams and long-edge graphs and then gave a natural decomposition of long-edge graphs into "templates" (Fomin and Mikhalkin did not name the graphs they use; the terminology "long-edge graphs" was first introduced in [2].)

In this section, we first give relevant definitions for long-edge graphs, followed by the main linearity result (Theorem 2.6 of this paper and an important result on a related function (Lemma 2.7). We start with some basic combinatorial definitions and notation that will be used in the rest of the paper. $\mathbb{N}=$ $\{0,1,2, \ldots$,$\} is the set of nonnegative integers and \mathbb{P}=\{1,2,3, \ldots$,$\} is the set of positive integers.$ Given a positive integer $\ell$, we denote by $[\ell]$ the set $\{1,2, \ldots, \ell\}$.

Definition 2.1 A long-edge graph $G$ is a graph $(V, E)$ with a weight function $\rho$ satisfying the following conditions:

a) The vertex set $V=\mathbb{N}=\{0,1,2, \ldots\}$, and the edge set $E$ is finite.

b) Multiple edges are allowed, but loops are not.

c) The weight function $\rho: E \rightarrow \mathbb{P}$ assigns a positive integer to each edge.

d) There are no short edges, i.e., there's no edges connecting $i$ and $i+1$ with weight 1 .

For any long-edge graph $G$ and any $k \in \mathbb{N}$, we denote by $G_{(k)}$ the graph obtained by shifting all edges of $G$ to the right $k$ units, i.e., a weighted edge $\{i, j\}$ in $G$ becomes a weighted edge $\{i+k, j+k\}$ in $G_{(k)}$.

We often draw the vertices $0,1,2, \ldots$ of long-edge graphs from left to right and label each edge with its weight. Since only finitely many vertices have incident edges, we often omit most of irrelevant vertices when we draw long-edge graphs. See Figure 1 for three examples of long-edge graphs. In particular, graph $G_{2}$ is obtained by shifting graph $G_{1}$. More precisely, $G_{2}=\left(G_{1}\right)_{(3)}$.

An important family of long-edge graphs is templates. 

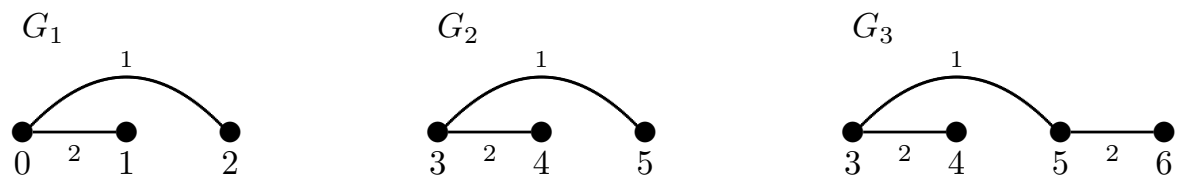

Fig. 1: Examples of long-edge graphs

Definition 2.2 A long-edge graph $\Gamma$ is a template if for any vertex $i: 1 \leq i \leq \operatorname{maxv}(\Gamma)-1$, there exists at least one edge $\{j, k\}$ satisfying $j<i<k$.

We say a long-edge graph $G$ is a shifted template if $G$ can be obtained by shifting a template; that is, if $G=\Gamma_{(k)}$ for some template $\Gamma$ and some nonnegative integer $k$.

Below we define some important statistics for long-edge graphs.

Definition 2.3 Given a long-edge graph $G=(V, E)$ equipped with weight function $\rho$ on the edge set $E$, we define the multiplicity of $G$ to be

$$
\mu(G):=\prod_{e \in E}(\rho(e))^{2}
$$

and the cogenus of $G$ to be

$$
\delta(G):=\sum_{e \in E}(l(e) \rho(e)-1),
$$

where for any $e=\{i, j\} \in E$ with $i<j$, we define $l(e):=j-i$. Note that any non-empty long-edge graph has positive cogenus.

We define $\operatorname{minv}(G)($ respectively, $\operatorname{maxv}(G))$ to be the smallest (respectively, largest) vertex of $G$ that has nonzero-degree. We then define the length of $G$, denoted by $l(G)$, to be $\operatorname{maxv}(G)-\operatorname{minv}(G)$.

Finally, we define for all $j$ :

$$
\begin{aligned}
& \lambda_{j}(G):=\text { sum of the weights of edges }\{i, k\} \text { with } i<j \leq k, \\
& \text { and } \bar{\lambda}_{j}(G):=\lambda_{j}(G)-\#(\text { edges in } G \text { connecting vertices } j-1 \text { and } j) .
\end{aligned}
$$

Example 2.4 See Table 1 for $\mu(\Gamma), \delta(\Gamma), l(\Gamma), \lambda(\Gamma)$ and $\bar{\lambda}(\Gamma)$ of templates of cogenus $\leq 2$.

Definition 2.5 Given a long-edge graph $G$, we say an ordered tuple $\left(G_{1}, \ldots, G_{i}\right)$ of (non-empty) longedge graphs is a partition of $G$ if the disjoint union of the (weighted) edge sets of $G_{1}, \ldots, G_{i}$ is the (weighted) edge set of $G$.

Fomin and Mikhalkin associate to each long-edge graph a statistic $\nu(G)$, and then give an enumerative formula for computing the Severi degree $N^{d, \delta}$ in terms of long-edge graphs using this statistic. We extend their definition and define two closely related statistics $P_{\boldsymbol{\beta}}(G)$ and $P_{\boldsymbol{\beta}}^{s}(G)$ for any given finite sequence $\boldsymbol{\beta}$, where $P_{(0,1, \ldots, d)}^{s}(G)$ is the same as $\nu(G)$ defined by Fomin and Mikhalkin. (The detailed definition of $P_{\boldsymbol{\beta}}(G)$ and $P_{\boldsymbol{\beta}}^{s}(G)$ is omitted.) We then consider the logarithmic versions of $P_{\boldsymbol{\beta}}(G)$ and $P_{\boldsymbol{\beta}}^{s}(G)$ :

$$
\Phi_{\boldsymbol{\beta}}(G):=\sum_{i \geq 1} \frac{(-1)^{i+1}}{i} \sum_{\left(G_{1}, \ldots, G_{i}\right)}\left(\prod_{j=1}^{i} P_{\boldsymbol{\beta}}\left(G_{j}\right)\right),
$$




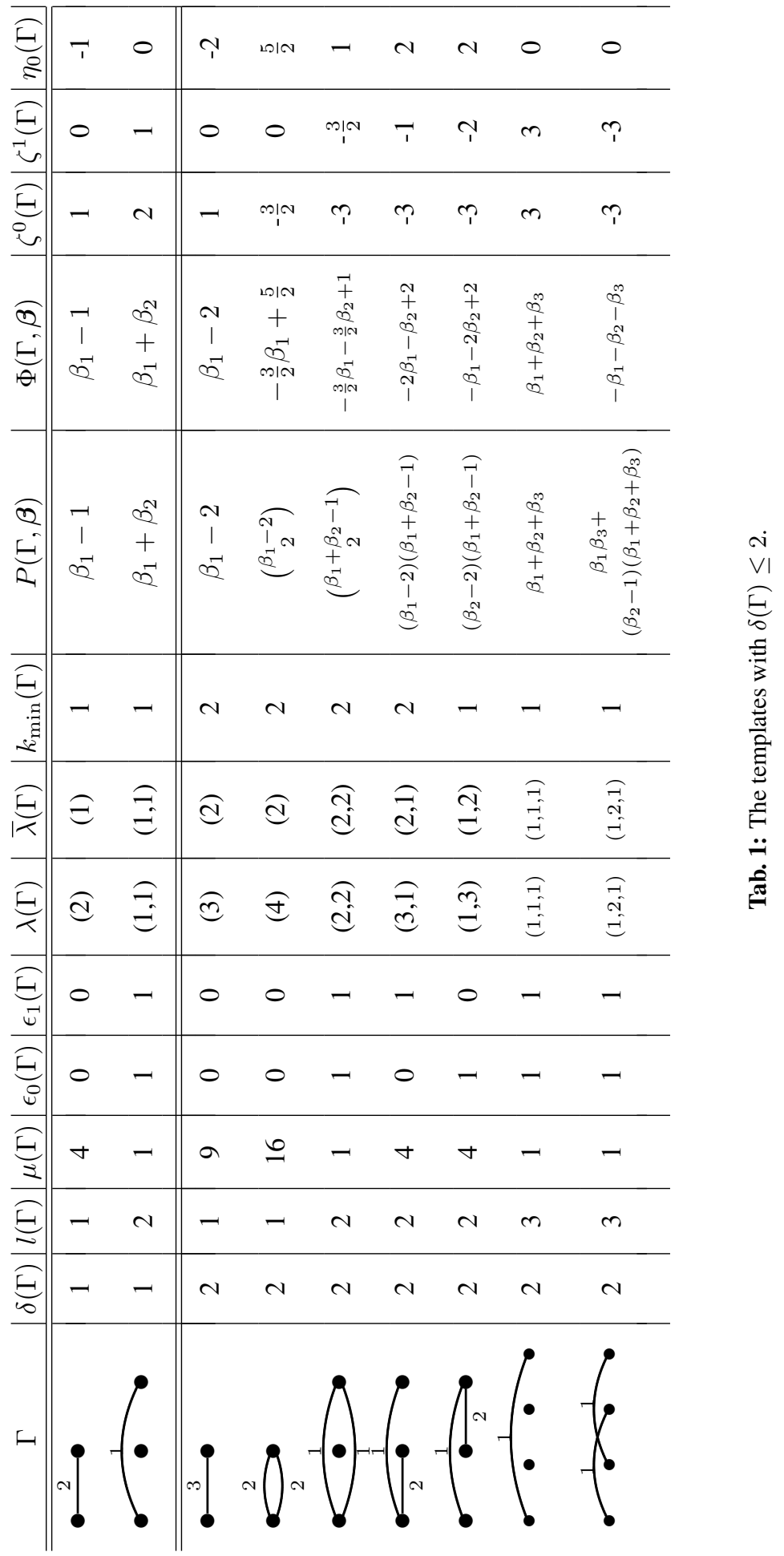




$$
\Phi_{\boldsymbol{\beta}}^{s}(G):=\sum_{i \geq 1} \frac{(-1)^{i+1}}{i} \sum_{\left(G_{1}, \ldots, G_{i}\right)}\left(\prod_{j=1}^{i} P_{\boldsymbol{\beta}}^{s}\left(G_{j}\right)\right)
$$

where both summations are over all the partitions of $G$.

Below is the main result of this paper.

Theorem 2.6 Suppose $G$ is a long-edge graph satisfying $\operatorname{maxv}(G) \leq M+1$. Then for any $\boldsymbol{\beta}=$ $\left(\beta_{1}, \ldots, \beta_{M+1}\right)$ satisfying $\beta_{j} \geq \bar{\lambda}_{j}(G)$ for all $j$, the values of $\Phi_{\boldsymbol{\beta}}(G)$ are given by a linear multivariate function $\Phi(G, \boldsymbol{\beta})$ in $\boldsymbol{\beta}$.

In addition to Theorem 2.6, which is a result on $\Phi_{\boldsymbol{\beta}}(G)$, we also have a fundamental result on $\Phi_{\boldsymbol{\beta}}^{s}(G)$, generalizing Proposition 4.4 of [2].

Lemma 2.7 Suppose $G$ is not a shifted template. Then $\Phi_{\boldsymbol{\beta}}^{s}(G)=0$.

Because the restriction of the length of this extended abstract, we are not able to include the complete proof of our main linearity result (Theorem 2.6) and Lemma2.7. Instead, we give a sketch of proof below.

Sketch of proof: The first part of the proof consists of a reformulation of our main results and two reduction steps. We start by introducing $\tau$-graphs, a generalization of long-edge graphs, and restate and generalize Theorem 2.6 and Lemma 2.7to versions using the language of $\tau$-graphs. The description of $\tau$ graphs enables us to consider generating functions of functions $\Phi_{\beta}^{s}$ and $\Phi_{\beta}$ in certain forms, which is used both to prove the generalized version of Lemma 2.7 and to reduce the problem of proving the generalized version of Theorem 2.6 to a result on generating functions. Next, we introduce another combinatorial object: $(\boldsymbol{\tau}, \boldsymbol{n})$-words, a special family of which, denoted by $S_{\boldsymbol{\tau}}(\boldsymbol{n}, \boldsymbol{t})$, has a reciprocity connection to $\tau$-graphs. Using this connection, we reduce our problem further to proving a result on the generating function of $S_{\boldsymbol{\tau}}(\boldsymbol{n}, \boldsymbol{t})$ (See Theorem 4.9 ).

In order to give a proof for Theorem 4.9 , we introduce a height function and a concept of irreducibility for $(\boldsymbol{\tau}, \boldsymbol{n})$-words. Using the height function, we describe an algorithm of finding the unique irreducible initial subword of a $(\boldsymbol{\tau}, \boldsymbol{n})$-word of prescribed height, which provides the main ingredient for proving a decomposition result on words in $S_{\boldsymbol{\tau}}(\boldsymbol{n}, \boldsymbol{t})$ and finishing the proof of Theorem 4.9. and thus finish the proof of our main linearity result (Theorem 2.6)

In Section 4, we will introduce the two important families of combinatorial objects mentioned above: $\boldsymbol{\tau}$-graphs and $(\boldsymbol{\tau}, \boldsymbol{n})$-words, and discuss how to obtain the linearity function asserted by Theorem 2.6 Before that, we discuss applications of our results.

\section{Applications of main results}

The proof of Theorem 2.6 is purely combinatorial and provides combinatorial objects to compute the coefficients of the linear function described in the theorem. We note that in 2012, the special case of Theorem 2.6 when $\boldsymbol{\beta}=(0,1,2, \ldots, d)$ was conjectured by Block, Colley and Kennedy. They have since given in [2] an independent proof of their conjecture. In fact, the original motivation of this paper was to prove their conjecture. However, the author noticed that the combinatorial approaches presented in this paper can be easily extended to prove our main result, leading to many more applications.

The first application of our theorem is that, as in [2], we can provide another proof for Corollary 1.2 by applying it to the logarithmic version of Fomin-Mikhalkin's formula for classical Severi degrees. 


\subsection{Analyzing Fomin-Mikhalkin's formula}

For the classical Severi degree, we only need to use $\boldsymbol{\beta}=(0,1,2, \ldots, d)$. Therefore, we give the following notation:

$$
\boldsymbol{v}(d):=(0,1,2, \ldots, d), \quad \forall d \in \mathbb{P} .
$$

Below is Fomin-Mikhalkin's formula for classical Severi degrees [7].

Theorem 3.1 (Fomin-Mikhalkin) The Severi degree $N^{d, \delta}$ is given by

$$
N^{d, \delta}=\sum_{G} \mu(G) P_{\boldsymbol{v}(d)}^{s}(G)
$$

where the summation is over all the long-edge graphs of cogenus $\delta$.

Recall that $\mathcal{N}(d)$ and $\mathcal{Q}(d)$ are defined as in 1.2 . Thus, $Q^{d, \delta}$ is the logrithmic version of $N^{d, \delta}$. Hence, with calculation, one obtains the logrithmic version of the above theorem:

$$
Q^{d, \delta}=\sum_{G} \mu(G) \Phi_{\boldsymbol{v}(d)}^{s}(G)
$$

where the summation is over all the long-edge graphs of cogenus $\delta$. This is the reason why we consider $\Phi_{\boldsymbol{\beta}}^{s}(G)$ (respectively, $\Phi_{\boldsymbol{\beta}}(G)$ ) the logarithmic version of $P_{\boldsymbol{\beta}}^{s}(G)$ (respectively, $P_{\boldsymbol{\beta}}(G)$ ).

One benefit of computing $Q^{d, \delta}$ instead of $N^{d, \delta}$ is that a lot of terms in (3.3) vanish. We have the following corollary to Lemma 2.7

Corollary 3.2 For $\delta \geq 1$,

$$
Q^{d, \delta}=\sum_{\Gamma} \mu(\Gamma) \sum_{k \in \mathbb{N}} \Phi_{\boldsymbol{v}(d)}^{s}\left(\Gamma_{(k)}\right),
$$

where the first summation is over all the templates of cogenus $\delta$.

It is an easy fact that for any fixed $\delta$, there are finitely many templates of cogenus $\delta$. Hence, the first summation in (3.4) is finite. It is not hard to see that the second summation in (3.4) has finitely many non-zero terms as well. Moreover, the linear function described in Theorem 2.6 is used for computing the second summation. Hence, we obtain another proof for Corollary 1.2 .

However, our techniques go further, providing a new method for computing $Q^{d, \delta}$ and $N^{d, \delta}$. We are also able to recover the threshold bound $d^{*} \leq \delta$ given by Block. We omit the details of these two results from this extended abstract. Furthermore, in the process of proving Corollary 1.2 , we discover combinatorial formulas involving the coefficients of the linear function described in Theorem 2.6 for computing the power series $A_{1}(t)$ and $A_{2}(t)$ of Proposition 1.1

\subsection{Determining $A_{1}(t)$ and $A_{2}(t)$}

For any power series $F(x)$, we denote by $\left[x^{\delta}\right] F(x)$ the coefficient of $x^{\delta}$ in $F(x)$.

Definition 3.3 Suppose $\Gamma$ is a template of length $\ell$, and $\Phi(\Gamma, \boldsymbol{\beta})=a_{0}+\sum_{j=1}^{\ell} a_{j} \beta_{j}$ be the polynomial assumed by Theorem 2.6 for $\Gamma$. We define

$$
\zeta^{i}(\Gamma):=\sum_{j=1}^{\ell}\left(\begin{array}{c}
j-1 \\
i
\end{array}\right) a_{j} \quad \text { for } i=0,1, \quad \text { and } \quad \eta_{0}(\Gamma):=a_{0} .
$$


See Table 1 for $\Phi(\Gamma, \boldsymbol{\beta}), \zeta^{0}(\Gamma), \zeta^{1}(\Gamma)$ and $\eta_{0}(\Gamma)$ of templates of cogenus $\leq 2$.

Corollary 3.4 The power series $A_{1}(t)$ and $A_{2}(t)$ of Proposition 1.1 are given by

$$
\begin{aligned}
& A_{1}(t)=\frac{1}{2} \sum_{\delta \geq 1}\left(\sum_{\Gamma: \delta(\Gamma)=\delta} \mu(\Gamma) \zeta^{0}(\Gamma)\right) t^{\delta} \\
& A_{2}(t)=\frac{1}{3} \sum_{\delta \geq 1}\left(\sum_{\Gamma: \delta(\Gamma)=\delta} \mu(\Gamma)\left(\frac{1}{2}\left(l(\Gamma)-\epsilon_{0}(\Gamma)-\epsilon_{1}(\Gamma)\right) \zeta^{0}(\Gamma)-\eta_{0}(\Gamma)\right)\right) t^{\delta} .
\end{aligned}
$$

(We omitted the definitions of $\epsilon_{0}(\Gamma)$ and $\epsilon_{1}(\Gamma)$, which were not very important.)

Remark 3.5 In [12] Lemma 7.1], the authors show that

$$
-\sum_{\Gamma} \mu(\Gamma)\left(l(\Gamma)-\epsilon_{0}(\Gamma)-\epsilon_{1}(\Gamma)\right) \zeta^{0}(\Gamma)=\sum_{\Gamma} \mu(\Gamma) \eta_{0}(\Gamma) .
$$

Applying this result, we obtain a simpler formula for the power series $A_{2}(t)$ :

$$
A_{2}(t)=-\frac{1}{2} \sum_{\delta \geq 1}\left(\sum_{\Gamma: \delta(\Gamma)=\delta} \mu(\Gamma) \eta_{0}(\Gamma)\right) t^{\delta}
$$

which only involves the constant term $\eta_{0}(\Gamma)$ in $\Phi(\Gamma, \boldsymbol{\beta})$.

Example 3.6 Applying Corollary 3.4 and using the data for $\zeta^{0}(\Gamma)$ in Table 1 we obtain the two lowest degree terms in $A_{1}(t)$ :

$$
\begin{aligned}
{[t] A_{1}(t) } & =\frac{1}{2}(4 \cdot 1+1 \cdot 2)=3 \\
{\left[t^{2}\right] A_{1}(t) } & =\frac{1}{2}\left(9 \cdot 1+16 \cdot\left(-\frac{3}{2}\right)+1 \cdot(-3)+4 \cdot(-3)+4 \cdot(-3)+1 \cdot 3+1 \cdot(-3)\right)=-21 .
\end{aligned}
$$

Thus, $A_{1}(t)=3 t-21 t^{2}+\cdots$.

Similarly, applying Corollary 3.4 and/or Remark 3.5 we can obtain the two lowest degree terms in $A_{2}(t)$. (Detailed calculation is omitted.)

$$
[t] A_{2}(t)=2, \quad\left[t^{2}\right] A_{2}(t)=-\frac{39}{2} .
$$

Hence, $A_{2}(t)=2 t-\frac{39}{2} t^{2}+\cdots$.

\subsection{More applications}

We finish this section with additional (and more important) application of our linearity result. The enumerative formula using labeled floor diagrams for Severi degrees introduced by Brugallé and Mikhalkin in [3, 4] works not only for complex projective planes $\mathbb{C P}^{2}$, but also for a more general family of (not necessarily smooth) toric surfaces coming from "h-transverse" polygons. In joint work with Osserman [12], we apply Theorem 2.6 to this family of toric surfaces and obtain universal polynomial results having a close connection to the Göttsche-Yau-Zaslow formula. Furthermore, results in [12] provide a simpler combinatorial formula for computing $A_{2}(t)$ (as in Remark 3.5 ) as well as a combinatorial formula for 
computing $A_{3}(t)-A_{4}(t)$ (where $A_{i}(t)$ 's are the power series described in Proposition 1.1). There are four power series involved in the Göttsche-Yau-Zaslow formula. Although two of the four power series, which are often referred to as $B_{1}(q)$ and $B_{2}(q)$, are not explicitly identified, their terms can be computed by using the recursive formula of [5] for the classical Severi degrees $N^{d, \delta}$ and applying interpolation methods as soon as the threshold $d^{*}$ of the polynomiality of $N^{d, \delta}$ is known. Assuming the threshold bound $d^{*} \leq\lceil\delta / 2\rceil+1$, Göttsche [8, Remark 2.5] has calculated $B_{1}(q)$ and $B_{2}(q)$ up to degree 28. Since $A_{2}(t)$ and $A_{3}(t)-A_{4}(t)$ determine $B_{2}(q)$ and $B_{1}(q)$ respectively, the paper [12] provides combinatorial methods for computing $B_{1}(q)$ and $B_{2}(q)$ directly without using interpolation.

\section{Computing the linear function}

In Subsection 3.2, we have described how to use coefficients of the linear function $\Phi(G, \boldsymbol{\beta})$ asserted by Theorem 2.6 to compute the power series $A_{1}(t)$ and $A_{2}(t)$ of Proposition 1.1. Therefore, it is important to understand how one can obtain these coefficients. In this section, we will introduce the combinatorial objects mentioned in the sketch of proof for Theorem 2.6 at the end of Section 2 and discuss results that are relevant for obtaining $\Phi(G, \boldsymbol{\beta})$. All the results given here will be stated without proofs.

We start by giving more notation that will be useful for the rest of the paper. We use $\mathbf{0}$ and $\mathbf{1}$ to denote vectors of all zeros and all ones respectively. Sometimes, we won't specify the dimensions of the vectors, which the readers should be able to figure out from the context. We define $\left(\mathbb{N}^{m}\right)^{*}:=\mathbb{N}^{m} \backslash \mathbf{0}$ to be the set of all vectors of $m$ nonnegative integers except the zero vector $\mathbf{0}$. For any $\boldsymbol{n}=\left(n_{1}, \ldots, n_{m}\right) \in \mathbb{N}^{m}$, we define

$$
\boldsymbol{x}^{\boldsymbol{n}}:=x_{1}^{n_{1}} x_{2}^{n_{2}} \cdots x_{m}^{n_{m}}, \quad(-1)^{\boldsymbol{n}}:=(-1)^{\sum_{i=1}^{m} n_{i}} .
$$

Hence, we can write $(-x)^{n}$ for $(-1)^{n} x^{n}$.

\section{1 -graphs: an alternative way of defining (long-edge) graphs}

Each edge $e$ of a (long-edge) graph $G$ contains two pieces of information: its weight $\rho(e)$ and its adjacent vertices. We use the set $I(e)=\{a+1, a+2, \ldots, b\}$ to represent the edge $\{a, b\}$ with $a<b$. In this case, we say $e$ is of type $(I(e), \rho(e))$. We will generalize this representation of describing an edge by a set and a number, and use it to define an abstract structure, which we call $\tau$-graph.

Definition 4.1 $A$ (generalized) edge of type $\tau=(I, \rho)$ consists of a finite subset $I$ of $\mathbb{N}$ and a positive integer $\rho$. We call $I$ the support of the edge, and $\rho$ is called its weight. An ordinary edge is one in which I is a set of consecutive integers $\{a+1, a+2, \ldots, b\}$.

Note that we have the concept of "head" and "tail" for an ordinary edge. However, we do not have this concept for a generalized edge.

Definition 4.2 Fixing a positive integer $m$, let $I_{1}, \ldots, I_{m}$ be subsets of $\mathbb{N}$ and $\rho_{1}, \ldots, \rho_{m} \in \mathbb{P}$. For each $1 \leq i \leq m$, let $\tau_{i}=\left(I_{i}, \rho_{i}\right)$. We may assume $\tau_{1}, \ldots, \tau_{m}$ are distinct. Let $\boldsymbol{\tau}=\left(\tau_{1}, \ldots, \tau_{m}\right)$.

For any $\boldsymbol{n}=\left(n_{1}, \ldots, n_{m}\right) \in \mathbb{N}^{m}$, we denote by $G_{\boldsymbol{\tau}}(\boldsymbol{n})$ the abstract structure that contains the following information:

- vertex set: $\mathbb{N}$;

- generalized edge set that consists of $n_{i}$ edges of type $\tau_{i}$ for each $1 \leq i \leq m$.

We call such an abstract structure a $\boldsymbol{\tau}$-graph. 
Remark 4.3 Note that if we require $\boldsymbol{\tau}=\left(\tau_{1}, \ldots, \tau_{m}\right)$ to satisfy that for each $1 \leq i \leq m$,

1. the set $I_{i}$ is a set of consecutive integers, and

2. the product $\rho_{i}\left|I_{i}\right|$ is greater than 1 ,

we can consider $G_{\boldsymbol{\tau}}(\boldsymbol{n})$ a long-edge graph. Hence, $\boldsymbol{\tau}$-graphs generalize long-edge graphs.

Strictly speaking, without condition (1), a $\tau$-graph is not a graph in the usual sense. In fact, we do not want to treat $\boldsymbol{\tau}$-graphs as graphs. The readers should just consider $\boldsymbol{\tau}$-graphs as abstract structures with prescribed data.

Example 4.4 1. Suppose $m=1$ and $\tau=\left(\tau_{1}\right)=((I, \rho))$, where $I=\{1\}$ and $\rho \in \mathbb{P}$. Then $G_{\tau}(n)$ is the graph with $n$ edges of weight $\rho$ connecting vertices 0 and 1 .

2. Suppose $m=2$ and $\boldsymbol{\tau}=\left(\tau_{1}, \tau_{2}\right)=\left(\left(I_{1}, \rho_{1}\right),\left(I_{2}, \rho_{2}\right)\right)$, where $I_{1}=\{1\}, I_{2}=\{1,2\}$ and $\rho_{1}, \rho_{2} \in \mathbb{P}$. Then $G_{\tau}\left(n_{1}, n_{2}\right)$ is the graph with $n_{1}$ edges of weight $\rho_{1}$ connecting 0 and 1 and $n_{2}$ edges of weight $\rho_{2}$ connecting vertices 0 and 2 .

\section{$4.2(\boldsymbol{\tau}, \boldsymbol{n})$-words}

For this part, we fix $\boldsymbol{\tau}=\left(\tau_{1}, \ldots, \tau_{m}\right)$, where $\tau_{i}=\left(I_{i}, \rho_{i}\right)$, and fix a positive integer $\ell$ such that $\ell$ is (greater than or) equal to the largest integer appearing in $I_{1}, \ldots, I_{m}$.

Definition 4.5 Fix $\boldsymbol{n} \in \mathbb{N}^{m}$. A $(\boldsymbol{\tau}, \boldsymbol{n})$-word is an ordered tuple of $\ell$ words $\left(w_{1}, \ldots, w_{\ell}\right)$ satisfying the following conditions:

a) Each $w_{j}$ is a sequence of letters chosen from $s_{0}, s_{1}, \ldots, s_{m}$ where repetition is allowed.

b) For each $1 \leq i \leq m$, the total number of $s_{i}$ appearing in all the words is $n_{i}$.

c) For each $1 \leq i \leq m$, the letter $s_{i}$ can only occur in words $w_{j}$ if $j \in I_{i}$.

Definition 4.6 Given a $(\boldsymbol{\tau}, \boldsymbol{n})$-word $\boldsymbol{w}=\left(w_{1}, \ldots, w_{\ell}\right)$, we define its height to be $h(\boldsymbol{w})=\left(h_{1}, \ldots, h_{\ell}\right)$ $=\left(h_{1}(\boldsymbol{w}), \ldots, h_{\ell}(\boldsymbol{w})\right)$, where $h_{j}=h_{j}(\boldsymbol{w})$ is defined by

$$
\begin{aligned}
h_{j}=h_{j}(\boldsymbol{w})= & (-1) \cdot \#\left(\text { letter } s_{0} \text { 's in } w_{j}\right)+\sum_{i: j \in I_{i}}\left(\rho_{i}-1\right) \cdot \#\left(\text { letter } s_{i} \text { 's in } w_{j}\right) \\
& +\sum_{i: j \in I_{i}} \rho_{i} \cdot \#\left(\text { letter } s_{i} \text { 's appearing in words other than } w_{j}\right) \\
= & (-1) \cdot \#\left(\text { letter } s_{0} \text { 's in } w_{j}\right)+\sum_{i: j \in I_{i}}\left(\rho_{i} \cdot \#\left(\text { letter } s_{i} \text { 's in } \boldsymbol{w}\right)-\#\left(\text { letter } s_{i} \text { 's in } w_{j}\right)\right) .
\end{aligned}
$$

We refer to $h$ as the height function.

We now consider a special family of $(\boldsymbol{\tau}, \boldsymbol{n})$-words.

Definition 4.7 Fixing $\boldsymbol{n} \in \mathbb{N}^{m}$, for any $\boldsymbol{t} \in \mathbb{N}^{\ell}$, we denote by $S_{\boldsymbol{\tau}}(\boldsymbol{n}, \boldsymbol{t})$ the set of all the $(\boldsymbol{\tau}, \boldsymbol{n})$-words $\boldsymbol{w}$ with $h(\boldsymbol{w})=-\boldsymbol{t}$.

We need the following concept of irreducibility.

Definition 4.8 Let $\boldsymbol{t} \in \mathbb{N}^{\ell}$ and $\boldsymbol{h}=-\boldsymbol{t}$. (So $\boldsymbol{h} \in\left(\mathbb{Z}_{\leq 0}\right)^{\ell}$.) Suppose the word $\boldsymbol{w}$ has height $\boldsymbol{h}$. (This means that $\boldsymbol{w} \in S_{\boldsymbol{\tau}}(\boldsymbol{n}, \boldsymbol{t})$ for some $\boldsymbol{n} \in \mathbb{N}^{m}$.) We say $\boldsymbol{w}$ is irreducible if it does not have a proper initial subword that also has height $\boldsymbol{h}$.

We denote by $S_{\boldsymbol{\tau}}^{\mathrm{irr}}(\boldsymbol{n}, \boldsymbol{t})$ the set of all $(\boldsymbol{\tau}, \boldsymbol{n})$-words that have height $\boldsymbol{h}=-\boldsymbol{t}$ and are irreducible. 
Fixing $\boldsymbol{n} \in \mathbb{N}^{m}$, we define the generating function of for $\left|S_{\boldsymbol{\tau}}(\boldsymbol{n}, \boldsymbol{t})\right|$ and $\left|S_{\boldsymbol{\tau}}^{\text {irr }}(\boldsymbol{n}, \boldsymbol{t})\right|$ :

$$
\begin{aligned}
& \mathcal{S}_{\boldsymbol{\tau}, \boldsymbol{t}}(\boldsymbol{x}):=\sum_{\boldsymbol{n} \in \mathbb{N}^{m}}\left|S_{\boldsymbol{\tau}}(\boldsymbol{n}, \boldsymbol{t})\right| \boldsymbol{x}^{\boldsymbol{n}}=1+\sum_{\boldsymbol{n} \in\left(\mathbb{N}^{m}\right)^{*}}\left|S_{\boldsymbol{\tau}}(\boldsymbol{n}, \boldsymbol{t})\right| \boldsymbol{x}^{\boldsymbol{n}}, \\
& \mathcal{S}_{\boldsymbol{\tau}, \boldsymbol{t}}^{\text {irr }}(\boldsymbol{x}):=\sum_{\boldsymbol{n} \in \mathbb{N}^{m}}\left|S_{\boldsymbol{\tau}}^{\operatorname{irr}}(\boldsymbol{n}, \boldsymbol{t})\right| \boldsymbol{x}^{\boldsymbol{n}}=1+\sum_{\boldsymbol{n} \in\left(\mathbb{N}^{m}\right)^{*}}^{\operatorname{irr}}(\boldsymbol{n}, \boldsymbol{t}) \mid \boldsymbol{x}^{\boldsymbol{n}} .
\end{aligned}
$$

The following is the key result for $(\boldsymbol{\tau}, \boldsymbol{n})$-words.

Theorem 4.9 Let $\boldsymbol{e}_{j}$ denotes the jth elementary vector of $\mathbb{R}^{\ell}$. Then for any $\boldsymbol{t} \in \mathbb{Z}^{\ell}$, we have

$$
S_{\boldsymbol{\tau}, \boldsymbol{t}}(\boldsymbol{x})=\left(\mathcal{S}_{\boldsymbol{\tau}, \boldsymbol{e}_{1}}^{\mathrm{irr}}(\boldsymbol{x})\right)^{t_{1}} \cdots\left(\mathcal{S}_{\boldsymbol{\tau}, \boldsymbol{e}_{\ell}}^{\mathrm{irr}}(\boldsymbol{x})\right)^{t_{\ell}} \cdot \mathcal{S}_{\boldsymbol{\tau}, \mathbf{0}}(\boldsymbol{x})
$$

\subsection{Determining $\Phi(G, \boldsymbol{\beta})$}

Finally, we will describe how to use the results presented above to compute $\Phi(G, \boldsymbol{\beta})$.

Lemma 4.10 Suppose $G$ is a long-edge graph that can be described as $G_{\boldsymbol{\tau}}(\boldsymbol{n})$, a $\boldsymbol{\tau}$-graph. Assume

$$
\log \mathcal{S}_{\boldsymbol{\tau}, \boldsymbol{e}_{j}}^{\mathrm{irr}}(\boldsymbol{x})=\sum_{\boldsymbol{n} \in\left(\mathbb{N}^{m}\right)^{*}} f^{(j)}(\boldsymbol{n}) \boldsymbol{x}^{\boldsymbol{n}} \quad \forall j, \quad \text { and } \quad \log \mathcal{S}_{\boldsymbol{\tau}, \mathbf{0}}(\boldsymbol{x})=\sum_{\boldsymbol{n} \in\left(\mathbb{N}^{m}\right)^{*}} h(\boldsymbol{n}) \boldsymbol{x}^{\boldsymbol{n}} .
$$

Then the linear function of $G$ asserted by Theorem 2.6 is

$$
\Phi(G, \boldsymbol{\beta})=(-1)^{\boldsymbol{n}}\left(-\sum_{j=1}^{\ell} f^{(j)}(\boldsymbol{n}) \beta_{j}+\left(h(\boldsymbol{n})-\sum_{j=1}^{\ell} f^{(j)}(\boldsymbol{n})\right)\right)
$$

Example 4.11 We consider the simplest situation: $m=1$ and $\tau=\left(\tau_{1}\right)=((\{1\}, r))$ where $r \in \mathbb{P}$. For any $n \in \mathbb{P}, G_{\tau}(n)$ is the graph with $n$ edges connecting vertices 0 and 1 , each of weight $r$.

We let $\ell=1$ and consider the corresponding $(\tau, n)$-words. (Note that we are in 1-dimensional space, so $e_{1}=1$ and $\mathbf{0}=0$.) In order to apply Lemma 4.10 we need to find $\left|S_{\tau}^{\operatorname{irr}}(n, 1)\right|$ and $\left|S_{\tau}(n, 0)\right|$. Clearly, $S_{\tau}(n, 0)$ is the collection of all words $w$ that consists of $n$ copies of $s_{1}$ and $n(r-1)$ copies of $s_{0}$. Hence, $\left|S_{\tau}(n, 0)\right|=\left(\begin{array}{c}r n \\ n\end{array}\right)$. On the other hands, one can show that words in $S_{\tau}^{\text {irr }}(n, 1)$ are in one-to-one correspondence to r-ary trees with $n$ internal vertices, whose cardinality is well-known to be $\frac{1}{1+(r-1) n}\left(\begin{array}{c}r n \\ n\end{array}\right)$. Therefore,

$$
\mathcal{S}_{\tau, 1}^{\operatorname{irr}}(x)=\sum_{n \in \mathbb{N}} \frac{1}{1+(r-1) n}\left(\begin{array}{c}
r n \\
n
\end{array}\right) x^{n}, \quad \mathcal{S}_{\tau, 0}^{\operatorname{irr}}(x)=\sum_{n \in \mathbb{N}}\left(\begin{array}{c}
r n \\
n
\end{array}\right) x^{n} .
$$

Applying Lagrange inversion formula [14] Corollary 5.4.3] (details omitted), we are able to find the coefficients of logarithms of the above two generating functions and obtain:

$$
\log \mathcal{S}_{\tau, 1}^{\mathrm{irr}}(x)=\sum_{n \in \mathbb{N}} \frac{1}{r n}\left(\begin{array}{c}
r n \\
n
\end{array}\right) x^{n}, \quad \log \mathcal{S}_{\tau, 0}^{\mathrm{irr}}(x)=\sum_{n \in \mathbb{N}}\left(\frac{1}{r n}\left(\begin{array}{c}
r n \\
n
\end{array}\right)+\frac{1}{n} \sum_{i=0}^{n-1}\left(\begin{array}{c}
r n \\
i
\end{array}\right)(r-1)^{n-1-i}\right) x^{n} .
$$

Therefore, by Lemma 4.10

$$
\Phi\left(G_{\tau}(n), \beta\right)=\frac{(-1)^{n+1}}{n}\left(\frac{1}{r}\left(\begin{array}{c}
r n \\
n
\end{array}\right) \beta-\sum_{i=0}^{n-1}\left(\begin{array}{c}
r n \\
i
\end{array}\right)(r-1)^{n-1-i}\right) .
$$

Furthermore, if we let $r=2$ and $n=2, G_{\tau}(n)$ is the fourth template appearing in Table 1 The above formula gives $\Phi\left(G_{\tau}(n), \beta\right)=-\frac{1}{2}(3 \beta-5)$, agreeing with the table. 


\section{References}

[1] F. Block, Computing node polynomials for plane curves, Math. Res. Lett. 18 (2011), no. 4, 621-643. MR 2831831

[2] F. Block, S. J. Colley, and G. Kennedy, Computing Severi degrees with long-edge graphs, Bull. Braz. Math. Soc. (N.S.) 45 (2014), no. 4, 625-647. MR 3296184

[3] E. Brugallé and G. Mikhalkin, Enumeration of curves via floor diagrams, C. R. Math. Acad. Sci. Paris 345 (2007), no. 6, 329-334. MR 2359091

[4] _ Floor decompositions of tropical curves: the planar case, Proceedings of Gökova Geometry-Topology Conference 2008, Gökova Geometry/Topology Conference (GGT), Gökova, 2009, pp. 64-90. MR 2500574 (2011e:14111)

[5] L. Caporaso and J. Harris, Counting plane curves of any genus, Invent. Math. 131 (1998), no. 2, 345-392. MR 1608583 (99i:14064)

[6] P. Di Francesco and C. Itzykson, Quantum intersection rings, The moduli space of curves (Texel Island, 1994), Progr. Math., vol. 129, Birkhäuser Boston, Boston, MA, 1995, pp. 81-148. MR 1363054 (96k:14041a)

[7] S. Fomin and G. Mikhalkin, Labeled floor diagrams for plane curves, J. Eur. Math. Soc. (JEMS) 12 (2010), no. 6, 1453-1496. MR 2734349

[8] L. Göttsche, A conjectural generating function for numbers of curves on surfaces, Comm. Math. Phys. 196 (1998), no. 3, 523-533. MR 1645204 (2000f:14085)

[9] J. Harris, On the Severi problem, Invent. Math. 84 (1986), no. 3, 445-461. MR 837522

[10] S. L. Kleiman and V. V. Shende, On the Göttsche threshold, A celebration of algebraic geometry, Clay Math. Proc., vol. 18, Amer. Math. Soc., Providence, RI, 2013, With an appendix by Ilya Tyomkin, pp. 429-449. MR 3114950

[11] M. Kool, V. Shende, and R. P. Thomas, A short proof of the Göttsche conjecture, Geom. Topol. 15 (2011), no. 1, 397-406. MR 2776848 (2012c:14113)

[12] F. Liu and B. Osserman, Severi degrees on toric surfaces, Journal für die reine und angewandte Mathematik (Crelle's Journal), to appear.

[13] N. Qviller, The Di Francesco-Itzykson-Göttsche conjectures for node polynomials of $\mathbb{P}^{2}$, Internat. J. Math. 23 (2012), no. 4, 1250049, 19. MR 2903195

[14] R.P. Stanley, Enumerative combinatorics. Vol. 2, Cambridge Studies in Advanced Mathematics, vol. 62, Cambridge University Press, Cambridge, 1999, With a foreword by Gian-Carlo Rota and appendix 1 by Sergey Fomin. MR 1676282

[15] Y. J. Tzeng, A proof of the Göttsche-Yau-Zaslow formula, J. Differential Geom. 90 (2012), no. 3, 439-472. MR 2916043 